\title{
A convenient synthesis of some new 2-oxazolidone derivatives
}

\author{
Syed Sulaiman Hussaini, V. Sriraman and C. A. M. A. Huq* \\ P.G. and Research Department of Chemistry, The New College, Chennai-600 014, India \\ E-mail : sshussainichem@rediffmail.com
}

Manuscript received 29 October 2002, revised 26 May 2003, accepted 4 September 2003

\begin{abstract}
One of the general approaches to the synthesis of $\mathbf{2}$-oxazolidones involves the reaction of glycols with urea. The synthesis of
\end{abstract} some novel 2-oxazolidone derivatives $3,5 \mathrm{a}$ and $5 \mathrm{~b}$ by the reaction of 1,2 diols $2,4 \mathrm{a}$ and $4 \mathrm{~b}$ with urea have been reported.

Lunsford et al. ${ }^{1}$ have reported the synthesis of 5 aryloxymethyl-2-oxazolidone in fairly good yield. We report here the synthesis of some interesting 2 -oxazolidone derivatives $1,10,10$-trimethyl-6-phenyl-3-oxa-5-azatricyclo[5.2.1. $\left.0^{2,6}\right]$ decan-4-one (3), 3a,11b-di-1 ${ }^{1}$-naphthyl$3 a, 11 b$-dihydro-3H-phenanthro[9,10- $d]$ oxazol-2-one (5a) and $3 a, 11 b$-diphenyl-3a, $11 b$-dihydro- $3 H$-phenanthro $[9,10-$ $d]$ oxazol-2-one (5b) using the above reaction.

\section{Results and discussion}

Camphor was treated with selenium dioxide in the presence of acetic anhydride to give 2,3-bornanedione ${ }^{2}$. It was converted to the corresponding $\beta$-keto alcohol (1) by the addition of two equivalents of phenylmagnesium bromide in dry THF. Reduction of 1 with sodium borohydride gave the diol (2) which on heating with two equivalents of urea gave the 2-oxazolidone (3) (Scheme 1).
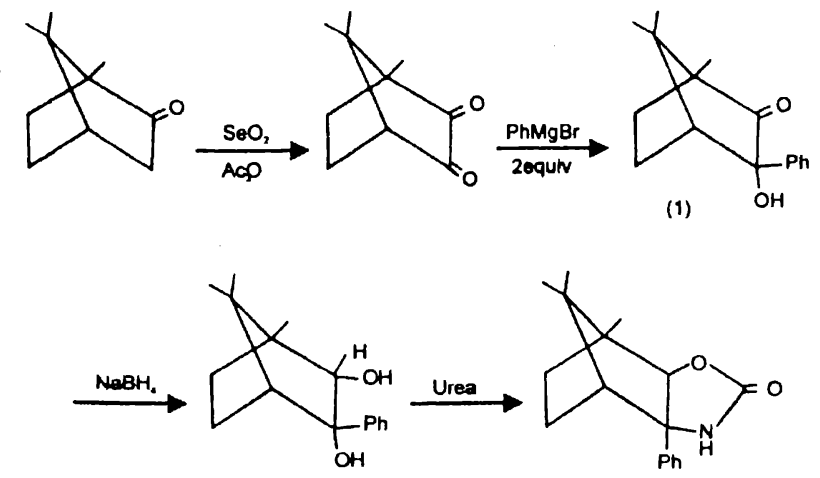

(2)

(3)

Scheme 1

The addition of four equivalents of arylmagnesium bromide to phenanthrene-9,10-dione in dry THF gave the corresponding diols $4 \mathbf{a}$ reported ${ }^{3}$ by us and $\mathbf{4 b}$, which on heating with two equivalents of urea gave the 2 -oxazolidones

(5a) and (5b) (Scheme 2).

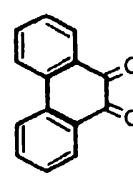

$y$

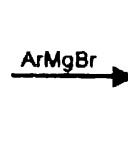

$(4 a, b)$

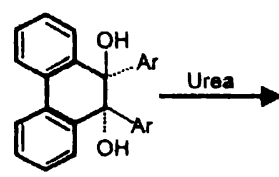

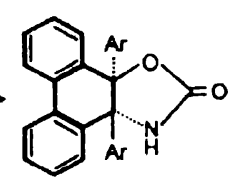

(5a, b)

$$
\begin{aligned}
& a=A r=1 \text { 'naphthyl } \\
& b=A r=p h e n y l
\end{aligned}
$$

Scheme 2

\section{Experimental}

All m.ps. are uncorrected. The purity of the compounds was checked by TLC on silica gel. IR spectra were recorded on a Perkin-Elmer 598 spectrophotometer, ${ }^{1} \mathrm{H}$ NMR spectra $\left(\mathrm{CDCl}_{3}\right)$ on a Varian EM 390 spectrometer and mass spectra on a Varian MatCH-7 spectrometer.

3-Hydroxy-1,7,7-trimethyl-3-phenyl-bicyclo[2.2.1]heptan-2-one (1): To a solution of phenylmagnesium bromide $(0.08 \mathrm{~mol}, 18.0 \mathrm{~g})$, (prepared from $2.0 \mathrm{~g}$ magnesium and $12.6 \mathrm{~g}$ bromobenzene) in dry THF $(200 \mathrm{ml})$ was added 2,3bornanedione $(0.04 \mathrm{~mol}, 6.64 \mathrm{~g})$. The mixture was refluxed for $3 \mathrm{~h}$ in dry nitrogen atmosphere and quenched with a saturated solution of ammonium chloride $(50 \mathrm{ml})$. The organic layer was separated. The aqueous layer was extracted with diethyl ether $(3 \times 20 \mathrm{ml})$. On removal of the solvent from the combined organic layer a viscous liquid $8.0 \mathrm{~g}$ was obtained. It was chromatographed over a column of silica gel. Elution with ethyl acetate : petroleum ether (1 : 20) gave a solid $(5.2 \mathrm{~g}, 85 \%)$, m.p. $105^{\circ}$ (Found : C, 78.61; $\mathrm{H}, 8.55 . \mathrm{C}_{16} \mathrm{H}_{20} \mathrm{O}_{2}$ requires : $\mathrm{C}, 78.69 ; \mathrm{H}, 8.60 \%$ ); $v_{\max }\left(\mathrm{CDCl}_{3}\right) 1600(\mathrm{C}=\mathrm{C}$, arom), $1740(\mathrm{C}=\mathrm{O}), 2800-3100$ $(\mathrm{C}-\mathrm{H}), 3550 \mathrm{~cm}^{-1}(\mathrm{OH}) ; \delta\left(\mathrm{CDCl}_{3}\right) 7.24(5 \mathrm{H}, \mathrm{s}, \mathrm{ArH}), 2.70$ $\left(1 \mathrm{H}, \mathrm{s}, \mathrm{OH}, \mathrm{D}_{2} \mathrm{O}\right.$ exchangeable $), 2.29(1 \mathrm{H}, \mathrm{t}, \mathrm{CH}), 1.64$ 
$\left(4 \mathrm{H}, \mathrm{m}, \mathrm{CH}_{2}\right), 0.9,1.0,1.1\left(9 \mathrm{H}, 3 \times \mathrm{s}, 3 \times \mathrm{CH}_{3}\right) ; \mathrm{m} / z 244$.

2,3-Dihydroxy-1,7,7-trimethyl-3-phenyl-bicyclo [2.2.1]heptane (2): The $\beta$-keto alcohol (1) was reduced with sodium borohydride to the diol (2) by the reported ${ }^{4}$ procedure, (yield 90\%), m.p. 157-159 (Found : C, 77.91; $\mathrm{H}, 8.90 . \mathrm{C}_{\mathrm{I} 6} \mathrm{H}_{22} \mathrm{O}_{2}$ requires : $\left.\mathrm{C}, 78.00 ; \mathrm{H}, 8.94 \%\right) ; v_{\max }$ $\left(\mathrm{CDCl}_{3}\right)$ 2850-3100(C-H), 3450 and $3600 \mathrm{~cm}^{-1}(\mathrm{OH}) ; \delta$ $\left(\mathrm{CCl}_{4}\right) 7.2(5 \mathrm{H}, \mathrm{m}, \mathrm{ArH}), 3.90\left(1 \mathrm{H}, \mathrm{d}, \mathrm{OH}, \mathrm{D}_{2} \mathrm{O}\right.$ exchangeable), $3.1(1 \mathrm{H}, \mathrm{d}, \mathrm{CH}), 2.7\left(1 \mathrm{H}, \mathrm{s}, \mathrm{OH}, \mathrm{D}_{2} \mathrm{O}\right.$ exchangeable), $2.1\left(4 \mathrm{H}, \mathrm{m}, \mathrm{CH}_{2}\right), 1.7(1 \mathrm{H}, \mathrm{t}, \mathrm{CH}), 0.9,1.0,1.1(9 \mathrm{H}$, $\left.3 \times \mathrm{s}, 3 \times \mathrm{CH}_{3}\right) ; \dot{m} / z 246$.

1,10,10-Trimethyl-6-phenyl-3-oxa-5-aza-tricyclo[5. 2.1.0 2,6 decan-4-one (3) : A mixture of diol $2(0.001 \mathrm{~mol}$, $0.246 \mathrm{~g})$ and urea $(0.002 \mathrm{~mol}, 0.120 \mathrm{~g})$ was heated at 180 $200^{\circ}$ for $5 \mathrm{~h}$. The reaction mixture was poured into water $(20 \mathrm{ml})$ and extracted with chloroform $(50 \mathrm{ml})$. It was dried over anhyd. $\mathrm{Na}_{2} \mathrm{SO}_{4}$. On removal of the solvent, a solid was obtained $(0.240 \mathrm{~g}, 90 \%), \mathrm{C}_{\mathrm{I}} \mathrm{H}_{21} \mathrm{NO}_{2}$, m.p. 123-124\%; $v_{\max }\left(\mathrm{CDCl}_{3}\right) 1740-1800(\mathrm{C}=\mathrm{O}), 3540 \mathrm{~cm}^{-1}(\mathrm{NH}) ; \delta$ $\left(\mathrm{CDCl}_{3}\right) 7.0(5 \mathrm{H}, \mathrm{s}, \mathrm{ArH}), 2.90(1 \mathrm{H}, \mathrm{s}, \mathrm{CH}), 2.2(4 \mathrm{H}, \mathrm{m}$, $\left.\mathrm{CH}_{2}\right), 1.7(1 \mathrm{H}, \mathrm{t}, \mathrm{CH}), 1.4\left(1 \mathrm{H}, \mathrm{s}, \mathrm{NH}_{2}, \mathrm{D}_{2} \mathrm{O}\right.$ exchangeable), $0.9,1.0,1.1\left(9 \mathrm{H}, 3 \times \mathrm{s}, 3 \times \mathrm{CH}_{3}\right) ; \mathrm{m} / \mathrm{z} 271$.

9,10-Di-1 ${ }^{1}$-naphthylphenanthrene-9,10-diol (4a): was synthesised by the reported procedure ${ }^{3}$.

3a,11b-Di-1'-naphthyl-3a,11b-dihydro-3Hphenanthro[9,10-d]oxazol-2-one (5a) : A mixture of diol $4 \mathbf{a}(0.001 \mathrm{~mol}, 0.464 \mathrm{~g})$ and urea $(0.002 \mathrm{~mol}, 0.120 \mathrm{~g})$ was rapidly heated at $180-200^{\circ}$ for $5 \mathrm{~h}$, then poured into water and extracted with chloroform $(50 \mathrm{ml})$. The chloroform layer was dried over anhyd. $\mathrm{Na}_{2} \mathrm{SO}_{4}$. On removal of the solvent, a solid was obtained $(0.200 \mathrm{~g}, 40 \%)$,m.p. $154-155^{\circ}$ (Found : $\mathrm{C}, 85.82 ; \mathrm{H}, 4.68 ; \mathrm{N}, 2.83 . \mathrm{C}_{35} \mathrm{H}_{23} \mathrm{NO}_{2}$ requires : $\mathrm{C}, 85.88$; $\mathrm{H}, 4.70 ; \mathrm{N}, 2.86 \%) ; v_{\max }\left(\mathrm{CDCl}_{3}\right) 1640-1700(\mathrm{C}=\mathrm{O}), 3520$ $\mathrm{cm}^{-1}(\mathrm{NH}) ; \delta\left(\mathrm{CDCl}_{3}\right) 8.1(2 \mathrm{H}, \mathrm{m}$, peri-protons), $7.3(2 \mathrm{H}$, $\mathrm{m}, \mathrm{ArH}), 2.0\left(\mathrm{lH}\right.$, br s, $\mathrm{NH}, \mathrm{D}_{2} \mathrm{O}$ exchangeable); $\mathrm{m} / z 489$.
9,10-Diphenylphenanthrene-9,10-diol (4b): It was synthesised from phenanthrene-9,10-diol $(0.025 \mathrm{~mol}, 7 \mathrm{~g})$ using phenylmagnesium bromide (prepared from $2.4 \mathrm{~g}$ magnesium and $15.6 \mathrm{~g}$ bromobenzene) in dry THF adapting the same procedure as for $\mathbf{4 a}$, (yield $6.0 \mathrm{~g}, 49 \%$ ), m.p. 171173 (Found : $\mathrm{C}, 85.66 ; \mathrm{H}, 5.47 . \mathrm{C}_{26} \mathrm{H}_{20} \mathrm{O}_{2}$ requires : $\mathrm{C}$, $85.71 ; \mathrm{H}, 5.49 \%) ; v_{\max }(\mathrm{KBr}) 1600(\mathrm{C}=\mathrm{C}$, arom $), 3520$ $\mathrm{cm}^{-1}(\mathrm{OH}) ; \delta\left(\mathrm{CDCl}_{3}\right) 7.2(18 \mathrm{H}, \mathrm{m}, \mathrm{ArH}), 4.1(2 \mathrm{H}, \mathrm{s}, \mathrm{OH}$, $\mathrm{D}_{2} \mathrm{O}$ exchangeable); $m / z 364$.

3a,11b-Diphenyl-3a,11b-dihydro-3H-phenanthrol9, 10-d Joxazol-2-one (5b) : A mixture ofdiol $\mathbf{4 b}(0.0005 \mathrm{~mol}$, $0.182 \mathrm{~g})$ and urea $(0.001 \mathrm{~mol}, 0.060 \mathrm{~g})$ was heated at 180 $200^{\circ}$ for $\sim 7 \mathrm{~h}$, then extracted with chloroform $(50 \mathrm{ml})$ and dried over anhyd. $\mathrm{Na}_{2} \mathrm{SO}_{4}$. On removal of the solvent, a white solid was obtained (yield $0.076 \mathrm{~g}, 40 \%$ ), m.p. 163$165^{\circ}$ (Found : C, 83.28; H, 4.86; N, 3.55. $\mathrm{C}_{27} \mathrm{H}_{19} \mathrm{NO}_{2}$ requires : C, 83.29; $\mathrm{H}, 4.88 ; \mathrm{N}, 3.59 \%) ; v_{\max }\left(\mathrm{CDCl}_{3}\right) 3520$ $\mathrm{cm}^{-1}(\mathrm{NH}) ; \delta\left(\mathrm{CDCl}_{3}\right) 7.1(18 \mathrm{H}, \mathrm{m}, \mathrm{ArH}), 1.6(\mathrm{lH}, \mathrm{s}, \mathrm{NH}$, $\mathrm{D}_{2} \mathrm{O}$ exchangeable); $m / z 389$.

\section{Acknowledgement}

The authors are thankful to SPIC Science Foundation, Chennai, R.S.I.C., I.I.T., Chennai, for providing the spectral data and the management of New College, Chennai, for facilities.

\section{References}

1. C. D. Lunsford, R. P. Mays and R. S. Murphey, J. Am. Chem. Soc., 1960, 82, 1166.

2. F. G. Mann and B. C. Saunders, "Practical Organic Chemistry", 4th. ed., ELBS and Longman, London, 1974, p. 147.

3. A. K. Rahiman, S. S. Hussaini and C. A. M. A. Huq, Curr. Sci., 2002, 82, 1138.

4. P. Dimroth, H. Pasedoch and K. Schefezik, Chem. Abstr., 1964, 60, 2934. 